\title{
REVIEW
}

\section{Scaffolded biosensors with designed DNA nanostructures}

\author{
Hao Pei ${ }^{1,2}$, Xiaolei Zuo ${ }^{1,2}$, Dun Pan ${ }^{1,2}$, Jiye Shi ${ }^{1,2,3}$, Qing Huang ${ }^{1,2}$ and Chunhai Fan ${ }^{1,2}$
}

In addition to its fundamental function as a genetic code carrier, the utilization of DNA in various material applications has been actively explored over the past several decades. DNA is intrinsically an excellent type of self-assembly nanomaterial owing to its predictable base-pairing, high chemical stability and the convenience it possesses for synthesis and modification.

Because of these unparalleled properties, DNA is widely used as excellent recognition elements in biosensors and as unique building blocks in nanodevices. A critical challenge in surface-based DNA biosensors lies in the reduced accessibility of target molecules to the DNA probes arranged on heterogeneous surfaces, especially when compared to probe-target recognition in homogeneous solutions. To improve the recognition abilities of these heterogeneous surface-confined DNA probes, much effort has been devoted to controlling the surface chemistry, conformation and packing density of the probe molecules, as well as the size and geometry of the surface. In this review, we aim to summarize the recent progress on the improvement of the probetarget recognition properties by introducing DNA nanostructure scaffolds. A range of new strategies have proven to provide a significantly enhanced range in the spatial positioning and the accessibility of the probes to the surface over previously reported linear structures. We will also describe the applications of DNA nanostructure scaffold-based biosensors. NPG Asia Materials (2013) 5, e51; doi:10.1038/am.2013.22; published online 14 June 2013

Keywords: biosensor; DNA nanostructure; interface; nanodevice

\section{INTRODUCTION}

Detection of nucleic acids (DNA or RNA) is an integral step in many applications, including in clinical diagnosis, environmental monitoring and antibioterrorism. ${ }^{1}$ With these applications in mind, significant efforts have been made to develop sensitive, selective, rapid and cost-effective DNA (or RNA) biosensors. In parallel, DNAbased devices have also attracted a significant, recent interest owing to their promising applications in nanoelectronics, ${ }^{2,3}$ biomolecular computations, ${ }^{4-8}$ cellular imaging ${ }^{9}$ and drug delivery. ${ }^{10-12}$ In a typical design of DNA biosensors and nanodevices, oligonucleotides are often used as the molecular recognition layer. Because DNA is comprised of only four bases with A-T and G-C pairing, DNA hybridization processes are highly predictable and can be finely tuned with a rational design of the sequence. In addition, DNA oligonucleotides are usually easy to design, chemically stable and cost-effective.

Understanding the physical structure of a DNA probe immobilized on a surface is critical in applications using DNA as a molecular recognition element. The properties of the DNA molecular recognition layer (such as selectivity, sensitivity and reproducibility) highly depend on the structure of the self-assembled DNA film. Modeling studies with thiolated DNA have demonstrated that efficient hybridization occurs in the well-controlled condition of surface-attached probes with large interprobe distances and upright conformations. ${ }^{13-24}$
However, constructing a reproducible DNA recognition layer with both of these properties is a critical challenge owing to unexpected surface adsorptions, disordered conformations, inconsistencies in grafting density and the flexibility of probe molecules. It is thus imperative to obtain an in-depth understanding of the factors that influence the structure of immobilized DNA layers. In this article, we aim to summarize the recent advances in this field, particularly in the evolution of using DNA probes from one-dimensional (1D) to twodimensional (2D) and ultimately to three-dimensional (3D) probes (Figure 1), as well as the application of these probes in developing DNA biosensors.

\section{D DNA PROBES}

Single-stranded (ss) oligonucleotides (1D DNA probes, typically constructed of 15-50 bases) are widely used in developing most DNA sensors and chips owing to their lack of secondary structure. 1D DNA probes modified with thiol groups at either the $3^{\prime}$ or $5^{\prime}$ terminus can readily self-assemble on gold surfaces; this self-assembly has become a paradigm for the design of DNA recognition layers. Thiolated DNA is expected to form self-assembled monolayers at $\mathrm{Au}$ surfaces via a single-point attachment, causing the probe to possess an upright orientation.

${ }^{1}$ Division of Physical Biology, Shanghai Institute of Applied Physics, Chinese Academy of Sciences, Shanghai, China; ${ }^{2}$ Bioimaging Center, Shanghai Synchrotron Radiation Facility, Shanghai Institute of Applied Physics, Chinese Academy of Sciences, Shanghai, China and ${ }^{3}$ UCB Pharma, Slough, UK 


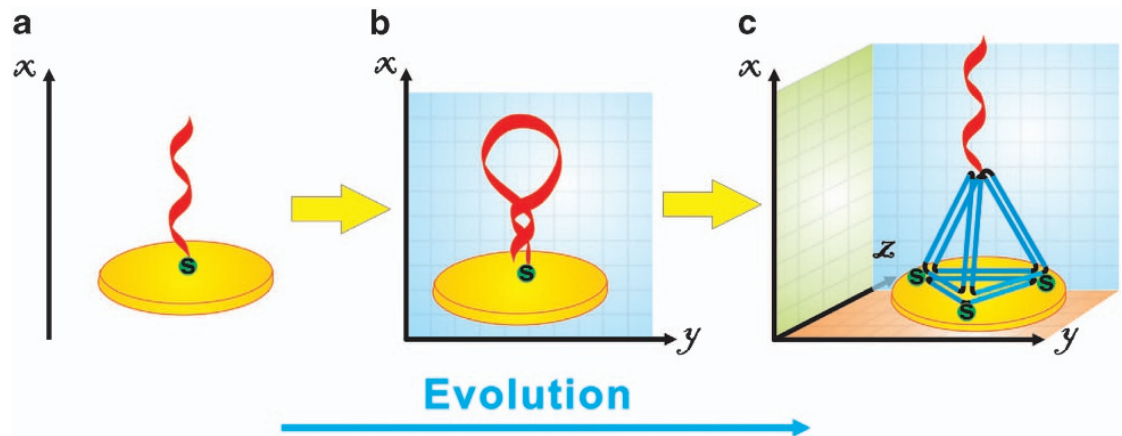

Figure 1 The evolution of surface-confined DNA probes from one-dimensional (1D) probes to two-dimensional (2D) and then three-dimensional (3D). Gold surfaces are anchored with thiolated DNA probes with (a) 1D single-stranded oligonucleotides (usually between 15 and 50 bases) without any secondary structure; (b) 2D DNA structure; and (c) 3D scaffolded DNA nanostructure.

However, because significant interactions exist between the DNA bases and the Au surface, in reality, DNA may lie down and adsorb to $\mathrm{Au}$ via multiple nitrogen atoms, ${ }^{25}$ which largely restricts the accessibility of the target DNA molecules (Figures 2a-c). In 1997, Herne and Tarlov ${ }^{17}$ provided evidence that thiolated single-stranded DNA (SH-ssDNA) is not only 'specifically' adsorbed onto the Au via the $\mathrm{Au}-\mathrm{S}$ bond but also 'nonspecifically' via $\mathrm{Au}-\mathrm{N}$ interactions. ${ }^{17}$ Moreover, X-ray photoelectron spectroscopy data revealed that such nonspecifically adsorbed ssDNA could not be removed, even with extensive rinsing or heating to $75^{\circ} \mathrm{C}$. An Au surface assembled with 25-base SH-ssDNA only has a film thickness of $3.3 \pm 0.2 \mathrm{~nm}$ in air, which is approximately $20 \%$ of the expected maximum thickness $(16 \mathrm{~nm})$, suggesting that the SH-ssDNA monolayer is not tightly packed and that the DNA chains are not oriented perpendicularly to the surface.

It was suggested that the attached DNA could extend further into the solution when either repulsive electrostatic fields ${ }^{26}$ were applied onto the surface or with a high surface density of DNA. ${ }^{27}$ By using high-resolution X-ray photoelectron spectroscopy and Fourier transform infrared spectroscopy, Petrovykh et al. ${ }^{20}$ characterized the structure of SH-ssDNA films on Au and showed that 1D DNA probes lay nearly flat on the substrate with multiple anchoring points at low densities. At high grafting densities, randomly coiled ssDNA probes were predominantly anchored via thiols and possibly one or two other bases. Further studies using near-edge X-ray absorption fine structure spectroscopy ${ }^{28}$ suggested that DNA bases in short probes tended to orient parallel to the surface. In contrast, long DNA probes formed disordered films similar to polyelectrolyte brushes. Clearly, short DNA probes may take upright conformations in densely packed films, which nevertheless prohibit efficient target accessibility.

Ideally, surfaces with a low density of DNA probes that possess the upright orientation should favor target hybridization. Tarlov and co-workers ${ }^{18}$ introduced a clever strategy that uses a coassembling 'helper' molecule, mercaptohexanol $(\mathrm{MCH})$, to solve this problem (Figure 2d). Treatment of the DNA-modified Au surface with $\mathrm{MCH}$ not only largely removes nonspecifically adsorbed DNA but also further projects the DNA probes out into the solution owing to the repulsion between the net negative dipole of the alcohol terminus and the negatively charged DNA backbones. These mixed ssDNA/ alkylthiol monolayers have been extensively characterized with neutron scattering, X-ray photoelectron spectroscopy, surface plasmon resonance and electrochemistry, which have all confirmed the favorable upright orientation of the DNA probes. ${ }^{29}$ The two-step sequential adsorption protocol has been extensively utilized in

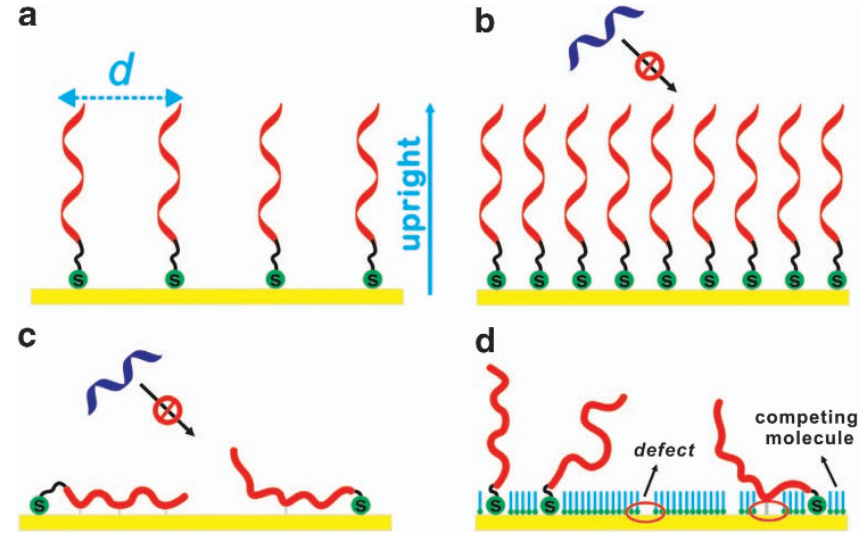

Figure 2 Impact of probe layer structure. (a) Schematic illustration of an ideal DNA recognition layer with large interprobe distances and upright conformation. (b) Densely packed DNA probes with an upright conformation, but resulting in poor hybridization efficiency that is due to steric hindrance. (c) Probes tend to lie flat on the surface owing to nonspecific adsorption in the sparse film. (d) Mixed single-stranded (ss)DNA/alkylthiol monolayers.

developing various types of DNA sensors and DNA-based devices. ${ }^{30}$ When coupled with signal amplification with enzymes or nanomaterials, high-sensitivity DNA sensors with detection limits down to the $\mathrm{pm}-\mathrm{fm}$ scale have been realized, which allows for the detection of a range of pathogenic or tumor targets. The use of $\mathrm{MCH}$ was later expanded with other diluent thiols. ${ }^{31,32}$ An example of this was a mixed ssDNA/oligo-ethylene glycol monolayer that was used to improve the protein resistance of the surface, which makes it broadly applicable in biological medium. ${ }^{33}$

The DNA probe density is a controlling factor for the efficiency of the target capture, as well as for the kinetics of the target/probe hybridization. $^{14,15}$ Gong and Levicky ${ }^{34}$ studied the hybridization behavior as a function of the ionic strength $\left(C_{\mathrm{B}}\right)$ and probe coverage $\left(S_{\mathrm{P}}\right)$ in the form of a diagram of hybridization regimens ${ }^{34}$ (Figure 3). They predicted that DNA hybridization is most favorable in the 'Langmuir' (L) regimen, which exists in the limits of sparse films where probes are so far apart that they do not interact. However, because significant lateral interactions exist in DNA films, even at the lowest densities, this $\mathrm{L}$ regimen was hardly reachable. Soft DNA polymers usually adopt disordered structures in the films, particularly at long base lengths. ${ }^{22,29,35}$ Recently, Josephs and $\mathrm{Ye}^{36}$ characterized the conformation of surface-tethered double-stranded DNA in a 
single-molecule view using electrochemical atomic force microscopy. They provided evidence that the conformation and dynamics of the tethered double-stranded DNA were far more complicated than expected from the view of ensemble measurements. The presence of aggregation patches significantly reduces target accessibility, which, nevertheless, could not be easily removed using $\mathrm{MCH}$ as the dilution molecule.

\section{D STRUCTURED DNA PROBES}

ssDNA strands with internal complementary sequences can form secondary structures (2D or pseudo-2D), such as hairpin, quadruplex and pseudoknot structures. Such relatively rigid and ordered structures may, in principle, prevent interstrand entanglement at the surface and/or increase the orderness of DNA layers.

In 2003, by attaching 2D DNA probes with a hairpin (stem-loop) structure at Au electrodes, Fan et al. ${ }^{37}$ designed a conceptually new electrochemical DNA (E-DNA) sensor strategy that exploits targetinduced conformational changes of 2D DNA structures (Figure 4a). The $3^{\prime}$ end and $5^{\prime}$ end of the stem are labeled with a thiol and an electroactive ferrocene, respectively. The hairpin probe was then self-assembled on Au via the $3^{\prime}$-alkanethiol. Target hybridization

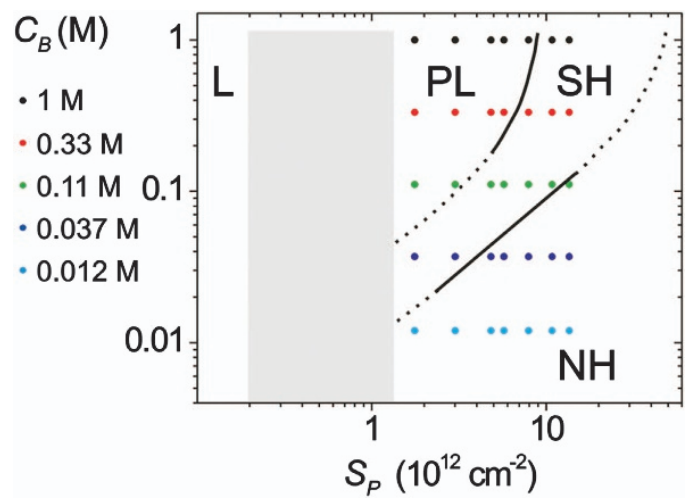

Figure 3 Diagram of surface hybridization regimes. $C_{B}$ : ionic strength; $S_{p}$ : probe coverage; non-hybridizing $(\mathrm{NH})$ regimen; suppressed hybridizing $(\mathrm{SH})$ regimen; pseudo-Langmuir $(\mathrm{PL})$ regimen; and a true 'Langmuir' $(\mathrm{L})$ regimen (adapted from Gong and Levicky ${ }^{34}$ ). alters the conformation of the probe, resulting in the opening of the stem and the extension of the loop into a linear duplex. In response to that extension, the distance between ferrocene and $\mathrm{Au}$ is increased, which leads to large variations in electron transfer and in turn produces measurable changes in electrochemical signals. This type of sensor can readily detect picomolar concentrations of target DNA without the need for exogenous reagents. This E-DNA sensor is rapid, portable and reusable, which is particularly useful in medical and military applications. By using this strategy, Lubin et al. ${ }^{43}$ successfully demonstrated an E-DNA sensor-based approach for detecting DNA authentication tags that are associated with paper or drugs. Lai et al. ${ }^{44}$ achieved the sequence-specific detection of unpurified amplification products of the gyrB gene of Salmonella typhimurium, which may open the path toward effective, field-portable sample-to-answer pathogen identification.

While the initial E-DNA sensor design has proven successful for the highly selective detection of target DNA in a wide range of mediums, the sensitivity is limited to picomolar concentrations, partially because one ferrocene label can only exchange one electron with the $\mathrm{Au}$ electrode. In 2005, Bockisch et al. ${ }^{45}$ reported a colorimetric method with stem-loop structured probes that relied on horseradish peroxidase (HRP) to transduce the conformation-associated signal. Given that one HRP can convert $\sim 10000$ reactions of hydrogen peroxide to water, the target binding-induced signal change could be greatly amplified. Liu et al. ${ }^{38}$ then developed a highly sensitive enzyme-based E-DNA sensor that pushed the detection limit down to low femtomolar concentrations, to nearly three orders of magnitude lower than the initial E-DNA setup ${ }^{38}$ (Figure $4 \mathrm{~b}$ ). Wei et $a l .{ }^{46}$ developed a similar sensor for electrochemical detection of salivary mRNA targets with $0.4 \mathrm{fm}$ sensitivity.

In an alternative approach, $\mathrm{Du}$ et al. ${ }^{47,48}$ proposed a fluorescent DNA sensor using DNA hairpins immobilized on the planar Au surface. Their study suggested that the hybridization efficiency is sensitive to the secondary structure of the hairpin, as well as to the distribution of the DNA hairpins on the substrate. Along this line, a range of fluorescent DNA sensors was developed using nanoscale metal surfaces. ${ }^{49-51}$ For example, we constructed multicolor nanobeacons by immobilizing three stem-loop probes labeled with different fluorophores on the surface of 15-nm gold nanoparticles (AuNPs). Three probes labeled with FAM, Cy5 and Rox were designed to target three types of tumor-suppressor genes (exon segments of
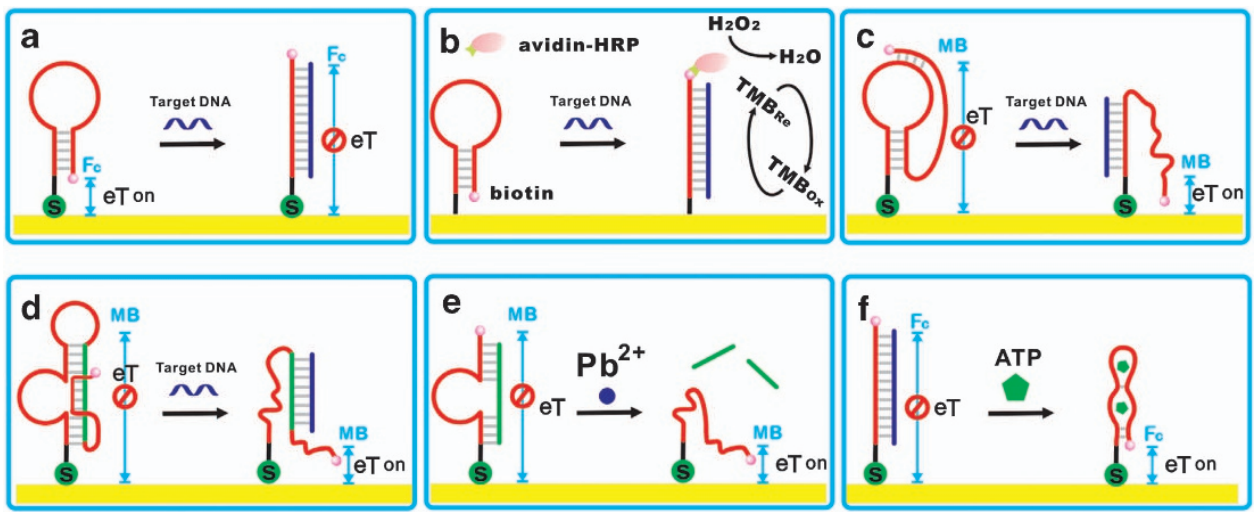

Figure 4 Two-dimensional (2D) DNA probe. (a) The stem-loop-structured probe design for an electrochemical DNA (E-DNA) sensor (adapted from Fan et $a l^{37}$ ). (b) A stem-loop-structured probe-based E-DNA sensor with enzyme amplification (adapted from Liu et al. ${ }^{41}$ ). (c) DNA probe with two stem-loop structures (adapted from Qu et al. ${ }^{51}$ ). (d) DNA probe with three stem-loop structures (adapted from Xiao et al. ${ }^{52}$ ). (e) DNAzyme-based 2D probe as an ion $\left(\mathrm{Pb}^{2+}\right)$ sensor (adapted from Plaxco et al. $\left.{ }^{53}\right)$. (f) DNA aptamer-based 2D probe as an ATP sensor (adapted from Zuo et al. ${ }^{54}$ ). HRP, horse radish peroxidase; $\mathrm{MB}$, multicolor beacons. 
p16, p21 and p53), and the nanobeacons could respond to the specific target with negligible crosstalk. ${ }^{52}$ It is interesting that AuNP-DNA conjugates can be efficiently taken up by cells. Jayagopal et al..$^{53}$ presented a strategy for live cell imaging of messenger RNA using DNA hairpin functionalized AuNPs, which shows high promise for real-time analysis of mRNA transport and processing in live cells.

In addition to stem-loop probes, it is also possible to design 2D probes with various different secondary structures. Xiao et al. ${ }^{54}$ designed a discontinuous duplex DNA probe for constructing a signal-on, label-free electronic DNA sensor, which utilized a stranddisplacement mechanism and achieved a sub-picomolar concentration detection limit. This improved detection limit is believed to be attributed to the enhanced rigidity of the sensing probe. Qu et al. ${ }^{39}$ designed another $2 \mathrm{D}$ probe using the pseudoknot structure that consists of two stem-loop structures in which a portion of each loop forms one strand of the stem of the other stem-loop (Figure 4c). They demonstrated that this probe was sufficiently stable for deployment directly in complex matrices, including in blood serum. Furthermore, Xiao et al. ${ }^{40}$ also used a triple-stem structure that could realize effective single-nucleotide polymorphism (SNP) detection at room temperature (Figure 4d). By comparing the thermodynamic analysis among single-, double- and triple-stem probes, they suggested that this probe took advantage of the large thermodynamic changes in enthalpy and entropy resulting from major conformational rearrangements in response to binding with the complementary target, which gave rise to exquisite sensitivity to single-base mismatches even in the complex medium of blood serum. ${ }^{40}$

It is worth mentioning that functional nucleic acids, such as DNAzymes or aptamers (RNA or DNA), can also be regarded as pseudo-2D probes. In 2007, Plaxco et al. ${ }^{41}$ used a $\mathrm{Pb}^{2+}$-requiring DNAzyme, the ' $8-17$ ' DNAzyme, as a probe for electrochemical detection of $\mathrm{Pb}^{2+}$ (Figure 4e). The probe consisted of a methylene blue-modified catalytic DNA strand hybridized to its complementary substrate oligonucleotide. The presence of $\mathrm{Pb}^{2}+$ induced cleavage of the complex and then disturbed the rigid structure of the complexes, which produced specific electrochemical signals. Zuo et al. ${ }^{42}$ designed an adenosine triphosphate (ATP) sensor using an anti-ATP aptamer. Their study verified that ATP could stabilize the tertiary aptamer structure at the surface and could responsively denature the initial aptamer duplex (Figure 4f). By incorporating a DNA aptamer into the three-way junction elements, Huang et al. ${ }^{55}$ reported a rationally designed probe architecture for the detection and quantification of thrombin, which is a plasma protein. With the same design principle, Ge et al. ${ }^{56}$ designed a contractile DNA duplex probe that incorporated two short, separated motifs of G/G mismatches that were flanked by Watson-Crick base-paired DNA. The presence of $\mathrm{K}^{+}$induced the transition of the duplex DNA to G-quadruplex and changed the charge-conducting properties of the DNA structure. Again, these 2D probes can be assembled on AuNPs and can be used to image molecular targets in live cells owing to the cellular uptake ability of AuNPs. Mirkin and co-workers ${ }^{57}$ attached an aptamer probe to AuNPs to develop an intracellular 'nanoflare' probe for quantification of small molecules or proteins in living systems.

Compared with $1 \mathrm{D}$ probes, $2 \mathrm{D}$ probes have shown superior properties as sensing elements in several aspects. First, the enhanced rigidity over the linear probes offers better ordered orientation of DNA probes on metal surfaces. Second, because of their internal hybridized nature, these $2 \mathrm{D}$ probes are inherently resistant to intraprobe interactions at the surface. Third, thermodynamic studies with 2D probes (single- or triple-stem structure) have demonstrated their capability to sensitively discriminate single-base mismatched DNA.
However, it should be noted that 2D probes are not sufficiently rigid to survive on highly crowded surfaces; hence, appropriate modulation of the surface density is still critically important for high-performance sensors. In addition, $\mathrm{MCH}$ is still required to help $2 \mathrm{D}$ structures stand upright at the surface, which makes the surface inevitably a heterogeneous one.

\section{D-STRUCTURED DNA PROBES}

It is critically important to control precisely the density and orientation of grafted DNA probes on the surface to minimize the lateral interactions between probes and to maximize the target accessibility. To this end, Pei et al. ${ }^{58}$ proposed a new concept to improve the probe-target recognition performance by introducing a 3D-structured DNA probe. As an example, a tetrahedron-shaped DNA nanostructure with a pendant DNA probe at one vertex and three thiol groups at the other three vertices was designed and used for the development of DNA sensors. This probe is expected to attach to the Au surface with the three-point attachment via the thiol-Au bond and to leave a free-standing probe at the top vertex. This tetrahedron-structured probe (TSP) is self-assembled with a probecontaining, 80-base oligonucleotide and three thiolated 55-base oligonucleotides. The formation of the TSP is essentially conducted in solution and is completed within only 2 min with a high yield of $\sim 85 \%$, as confirmed by non-denatured electrophoresis analysis. TSP is rapidly and firmly adsorbed at the surface of gold owing to the presence of the three thiol legs, a process that was monitored with a combination of surface-sensitive technologies, including quartz crystal microbalance, surface plasmon resonance and microcantilever. The probe-to-probe spacing of TSP is approximately $4 \mathrm{~nm}$, as estimated from the measured surface density of TSP $\left(4.8 \times 10^{12} \mathrm{TSP}_{\mathrm{cm}}{ }^{-2}\right)$. The pendant probe can selectively capture its complementary sequence, with high discrimination ability toward non-cognate sequences.

By using TSP-decorated Au electrodes, Pei et al. ${ }^{58}$ constructed an E-DNA sensor with a conventional sandwich detection strategy (Figure 5a). The electrochemical signal is produced via the substrate catalysis of HRP. Of significance, this non-optimized, proof-ofconcept sensor exhibits a 250-fold improvement in sensitivity ( $\sim 1 \mathrm{pM})$ compared with that of 1D probes with the similar HRPbased signal-transduction approach. To further improve the sensitivity, Wen et al. ${ }^{61}$ increased the interprobe distance using interfacial engineering and multienzyme amplification, which results in an extremely low detection limit of 10 am $(\sim 600$ molecules in $100 \mu \mathrm{l})$.

The presence of bulky tetrahedral structures not only avoids interprobe entanglement via spatially segregating pendant probes but also reduces the surface effects and places the probes in a solution-phase-like environment. The high mechanical rigidity of TSP allows it to stay at the Au surface with a highly ordered, upright orientation. Hence, the nanostructured surface has superior molecular accessibility to conventional ones. For example, Howorka and coworkers $^{62}$ reported that molecular recognition of receptors on such a surface was improved by an order of magnitude. Squires et al. ${ }^{63}$ also found that the TSP-based DNA sensor possesses remarkable selectivity toward single-base mismatches. In addition, diffusion and convection at the nanostructure interfaces are also expected to be higher than those at macroscopic ones. This 3D-structured platform also demonstrates a combination of other advantages. First, the presence of the three thiol legs greatly increases the stability of the surfaceconfined probes by approximately 5000 times compared with the mono-thiolated DNA, which is beneficial for constructing stable and robust biosensors. ${ }^{64}$ In addition, the 'helper' molecule $\mathrm{MCH}$ is not necessary in this case. Second, this TSP-modified surface is fully 

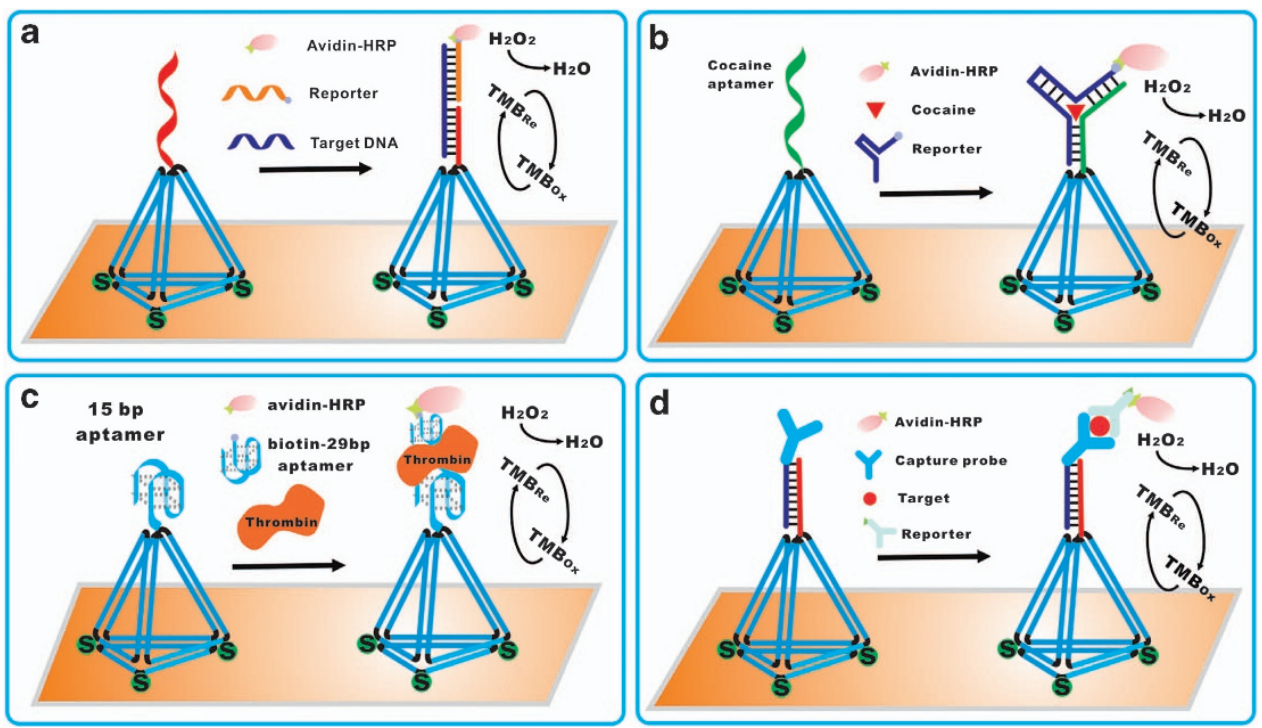

Figure 5 The tetrahedron-structured probe (TSP)-based biosensing platform for (a) DNA targets (adapted from Pei et al. ${ }^{58}$ ); (b) a small molecule, cocaine, with the split anticocaine aptamer (adapted from Wen et al. ${ }^{63}$ ); (c) a protein, thrombin, with a pair of antithrombin aptamers (adapted from Pei et al. ${ }^{58}$ ); and (d) immunological assays (adapted from Pei et al.66). HRP, horse radish peroxidase.

compatible with electrochemical interrogations. While the TSP layer is relatively thick $(\sim 6 \mathrm{~nm})$, the hollow structure of the TSP allows facile communications of electroactive species with the electrode. Third, the TSP-decorated surfaces can prevent nonspecific adsorption of proteins, which makes it possible to use directly TSP-based sensors in the serum for practical applications.

The TSP-based surface has proven to be a versatile platform for the detection of a broad range of biomolecules. The pedant probe can be replaced with various functional nucleic acids for biodetection. As a proof of concept, a split aptamer for a small molecule, cocaine, was incorporated to develop an electrochemical sensor for cocaine ${ }^{59}$ (Figure 5b). This sensor could detect a concentration of $33 \mathrm{~nm}$ cocaine in a complex media, exceeding the sensitivity of previously reported aptamer-based sensors by more than two orders of magnitude. In another approach, Pei et al. ${ }^{58}$ replaced the DNA probe with an antithrombin aptamer at the top of the tetrahedron, which formed a thrombin sensor that could detect a concentration as low as $100 \mathrm{pm}$ thrombin (Figure 5c). In addition, Ge et al. ${ }^{65}$ developed an electrochemical sensor for the genotyping of SNPs, and Bu et al. ${ }^{66}$ constructed a sensor for mercury ions using a mercury-specific oligonucleotide. To make this nanostructured surface more generally usable, Pei et al. ${ }^{60}$ introduced a DNA-directed antibody immobilization to convert the DNA recognition layer into a protein recognition one (Figure 5d). Antibodies can be easily and reversibly anchored onto this nanostructured surface via a DNA bridge. These immunosensors demonstrated superior sensitivity and selectivity over conventional ones.

Because surface-confined tetrahedra are highly rigid scaffolds, it is possible to place different dyes or redox molecules on the different bases of the structure to measure the distance-dependent energy transfer or charge transport. When a tetramethylrhodamine dye is placed at the top vertex, the fluorescence is intense; the fluorescence is observed to be weaker when the dye is attached at one of the bottom vertices that are close to the Au surface. This change in the strength of fluorescence is typical of Au-quenched fluorescence, which is due to the energy transfer distance. Similarly, Lu et al. ${ }^{67}$ conjugated either methylene blue or ferrocene redox molecules to different positions of the surface-confined DNA tetrahedron and studied the throughduplex and through-space DNA-mediated charge transport. Such interesting properties are particularly useful for the design of molecular sensors for external stimuli. For example, Pei et al. ${ }^{68}$ incorporated different functional nucleic acids into one or two edges of the tetrahedra, which made them dynamic structures that were responsive to different molecules. Because DNA tetrahedra can effectively enter mammalian cells without the help of a transfection reagent, ${ }^{9,10}$ this tetrahedron-based sensor can be used to image intracellular ATP in live cells.

\section{NANOSTRUCTURED DNA SUBSTRATES}

Self-assembled DNA nanostructures can also serve as the substrate for biosensing and bioassays (Figure 6). Compared with solid-state substrates (for example, gold or glass), DNA-based nanostructured substrates are essentially in solution and fully compatible with addressable DNA immobilization. This places the biological reactions in the homogeneous solution-like environment, which allows the reactions to occur faster than those that occur at heterogeneous interfaces.

Instead of using simple self-assembled DNA nanostructures, Ke et al. ${ }^{69}$ used a DNA origami ${ }^{70}$-based rectangular structure of $70 \times 100 \mathrm{~nm}^{2}$, which was appended with 20-base probes at predefined positions. This system could detect microRNAs (200 pм) in a high background of total cellular RNA. Because the probes are placed on the rectangular substrate with nanometer-scale precision, the site-specific effects of the probes could be explored with molecular resolution. However, the hybridization signal was only detectable with atomic force microscopy. Given that this tiny DNA origami chip might be easily scaled down to a sample volume comparable to that of single cells, it shows high promise for single-cell gene expression analysis.

This origami substrate of a rectangular shape is readable only when built-in 'index' oligonucleotides are present to break the symmetry. To overcome this problem, Zhang et al. ${ }^{71}$ reported the design of an 


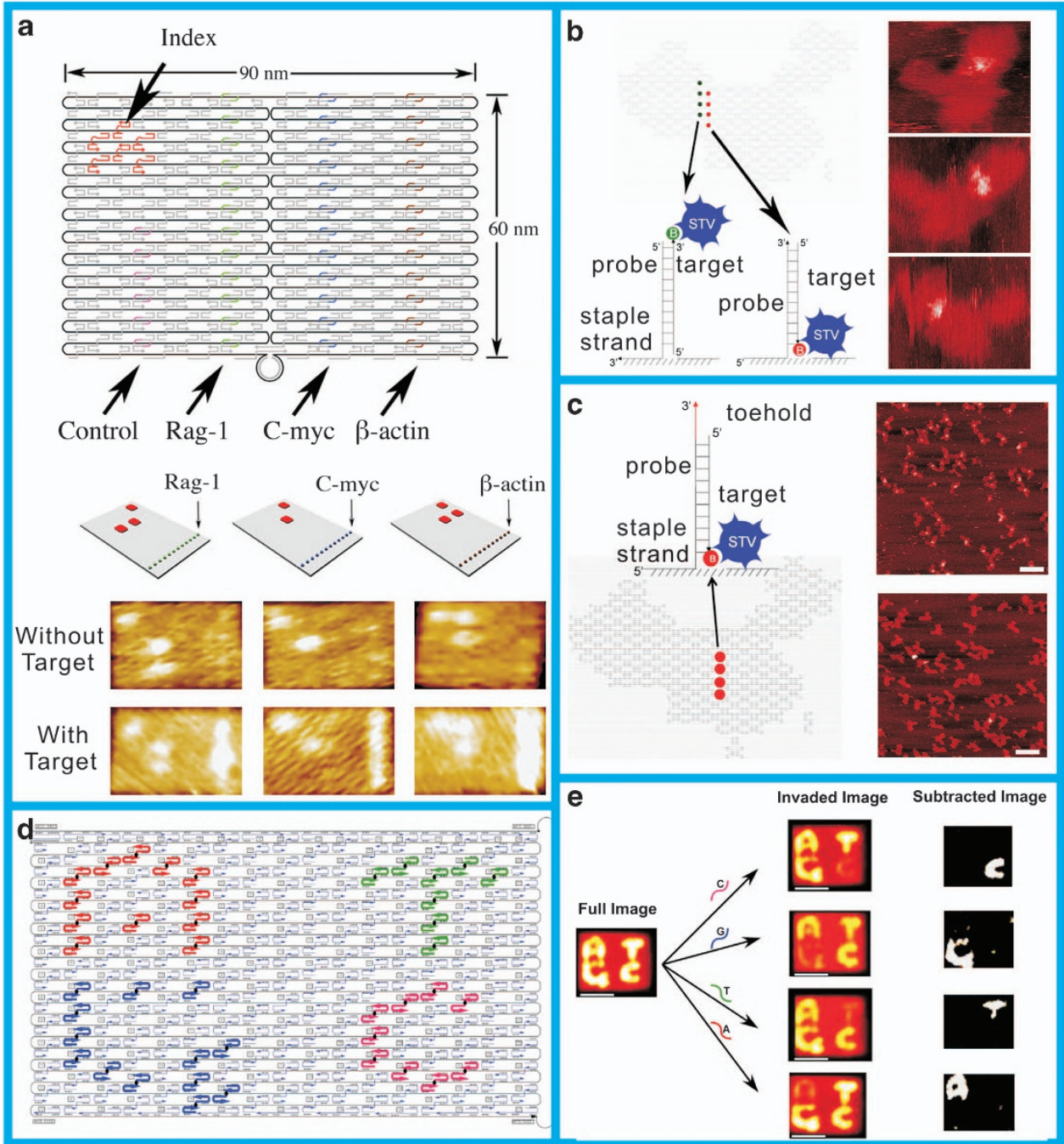

Figure 6 (a) A rectangular DNA origami chip for label-free detection of microRNAs (adapted from Ke et al. ${ }^{70}$ ). A map-shaped asymmetric DNA origami chip for the detection of (b) DNA hybridization and (c) single-nucleotide polymorphism (SNP) genotyping (adapted from Zhang et al. ${ }^{71,72}$ ); and (d and e) visual SNP genotyping on DNA origami with a symbolic display (adapted from Subramanian et al. ${ }^{73}$ ).

index-free nanoscale DNA chip by using an asymmetric DNA origami substrate with the shape of a Chinese map. The asymmetric nature of the map makes the position of each DNA probe fully spatially addressable under atomic force microscopy imaging. Zhang et al. ${ }^{72}$ also developed a chip for SNP genotyping on the DNA origami chip by introducing a toehold strand-displacement reaction that could effectively differentiate single-base mismatches at ambient temperature. Seeman and co-workers ${ }^{73}$ developed a visual DNA origami chip for SNP genotyping with a symbolic display. This DNA origami chip contains graphical representations of the four nucleotide alphabetic characters, A, T, G and C, and these patterns can be imaged with atomic force microscopy.

\section{CONCLUSION}

Along with the rapid emergence and development of biotechnology and nanotechnology, various DNA nanostructure scaffolds have been designed, characterized and exploited for a range of applications. ${ }^{74}$ In particular, we have observed the evolution of surface-confined DNA probes with rational design from $1 \mathrm{D}$ to $2 \mathrm{D}$ and then to $3 \mathrm{D}$, which greatly improves our ability to control the density, orientation and passivation of the surface. On the basis of these studies, a variety of electrochemical and optical biosensors have been constructed for the detection of DNA/RNA hybridization, SNP genotyping and microRNA biomarkers. Other types of 3D-structured DNA probes with different shapes and sizes should be explored in the future, which may provide additional advantages. Nevertheless, DNA sequence synthesis and modification form the major barrier for the design of 3D-structured DNA probes. Ideally, the availability of DNA sequences modified with functional biomolecules, for example, functional nucleic acids that can be readily folded into the desired DNA nanostructure, is necessary for the establishment of a generic platform for biosensing using 3D DNA probes. However, the synthesis of long DNA sequences ( $>100$ nucleotides) is not readily commercially available. In addition, extra functional biomolecules may influence the assembly of the DNA nanostructure. The purity of the assembled DNA nanostructure is another concern and relies on the improvement of amenable separation techniques, for example, electrophoresis or high-performance liquid chromatography.

More recently, emerging DNA nanotechnology offers the unprecedented ability to control the assembly of large and well-defined DNA 
nanostructures that may serve as substrates for site-specific immobilization of biomolecules. This control will provide new opportunities to perform chip-based multianalyte bioassays in homogeneous solutions. Parallel to these advances in biosensing, DNA nanostructure-based nanodevices have also attracted intense interests that have led to the development of nanorobots, nanocarriers for drug delivery and nanoscale computing devices. All of these advances rely on the active interdisciplinary cooperation among chemistry, physics, material science, biology and electronics, which will clearly have a bright future regarding diagnosis and therapy of cancers and other important diseases.

\section{ACKNOWLEDGEMENTS}

This work was supported by the Ministry of Science and Technology of China (2012CB932600 and 2013CB932803), the National Science Foundation of China (91123037) and the Chinese Academy of Sciences.

1 Heller, M. J. DNA microarray technology: devices, systems, and applications. Annu. Rev. Biomed. Eng. 4, 129-153 (2002).

2 Slinker, J. D., Muren, N. B., Renfrew, S. E. \& Barton, J. K. DNA charge transport over 34 nm. Nat. Chem. 3, 228-233 (2011).

3 Genereux, J. C. \& Barton, J. K. Mechanisms for DNA charge transport. Chem. Rev. 110, 1642-1662 (2010).

4 Willner, I., Shlyahovsky, B., Zayats, M. \& Willner, B. DNAzymes for sensing nanobiotechnology and logic gate applications. Chem. Soc. Rev. 37, 1153-1165 (2008).

5 Seelig, G., Soloveichik, D., Zhang, D. Y. \& Winfree, E. Enzyme-free nucleic acid logic circuits. Science 314, 1585-1588 (2006)

6 Qian, L. \& Winfree, E. Scaling up digital circuit computation with DNA strand displacement cascades. Science 332, 1196-1201 (2011).

7 Qian, L., Winfree, E. \& Bruck, J. Neural network computation with DNA strand displacement cascades. Nature 475, 368-372 (2011).

8 Pei, R. J., Matamoros, E., Liu, M. H., Stefanovic, D. \& Stojanovic, M. N. Training a molecular automaton to play a game. Nat. Nanotechnol. 5, 773-777 (2010).

9 Walsh, A. S., Yin, H. F., Erben, C. M., Wood, M. J. A. \& Turberfield, A. J. DNA cage delivery to mammalian cells. ACS Nano 5, 5427-5432 (2011).

10 Li, J., Pei, H., Zhu, B., Liang, L., Wei, M., He, Y., Chen, N., Li, D., Huang, Q. \& Fan, C. H. Self-Assembled multivalent DNA nanostructures for noninvasive intracellular delivery of immunostimulatory CpG oligonucleotides. ACS Nano 5, 8783-8789 (2011).

11 Douglas, S. M., Bachelet, I. \& Church, G. M. A logic-gated nanorobot for targeted transport of molecular payloads. Science 335, 831-834 (2012).

12 Lee, H., Lytton-Jean, A. K. R., Chen, Y., Love, K. T., Park, A. I., Karagiannis, E. D., Sehgal, A., Querbes, W., Zurenko, C. S., Jayaraman, M., Peng, C. G., Charisse, K., Borodovsky, A., Manoharan, M., Donahoe, J. S., Truelove, J., Nahrendorf, M., Langer, R. \& Anderson, D. G. Molecularly self-assembled nucleic acid nanoparticles for targeted in vivo siRNA delivery. Nat. Nanotechnol. 7, 389-393 (2012).

13 Georgiadis, R., Peterlinz, K. P. \& Peterson, A. W. Quantitative measurements and modeling of kinetics in nucleic acid monolayer films using SPR spectroscopy. J. Am. Chem. Soc. 122, 3166-3173 (2000).

14 Peterson, A. W., Heaton, R. J. \& Georgiadis, R. Kinetic control of hybridization in surface immobilized DNA monolayer films. J. Am. Chem. Soc. 122, 7837-7838 (2000).

15 Peterson, A. W., Heaton, R. J. \& Georgiadis, R. M. The effect of surface probe density on DNA hybridization. Nucleic Acids Res. 29, 5163-5168 (2001).

16 Peterson, A. W., Wolf, L. K. \& Georgiadis, R. M. Hybridization of mismatched or partially matched DNA at surfaces. J. Am. Chem. Soc. 124, 14601-14607 (2002).

17 Herne, T. M. \& Tarlov, M. J. Characterization of DNA probes immobilized on gold surfaces. J. Am. Chem. Soc. 119, 8916-8920 (1997).

18 Levicky, R., Herne, T. M., Tarlov, M. J. \& Satija, S. K. Using self-assembly to control the structure of DNA monolayers on gold: a neutron reflectivity study. J. Am. Chem. Soc. 120, 9787-9792 (1998)

19 Peterlinz, K. A., Georgiadis, R. M., Herne, T. M. \& Tarlov, M. J. Observation of hybridization and dehybridization of thiol-tethered DNA using two-color surface plasmon resonance spectroscopy. J. Am. Chem. Soc. 119, 3401-3402 (1997).

20 Petrovykh, D. Y., Kimura-Suda, H., Whitman, L. J. \& Tarlov, M. J. Quantitative analysis and characterization of DNA immobilized on gold. J. Am. Chem. Soc. 125, 5219-5226 (2003).

21 Steel, A. B., Herne, T. M. \& Tarlov, M. J. Electrochemical quantitation of DNA immobilized on gold. Anal. Chem. 70, 4670-4677 (1998).

22 Steel, A. B., Levicky, R. L., Herne, T. M. \& Tarlov, M. J. Immobilization of nucleic acids at solid surfaces: effect of oligonucleotide length on layer assembly. Biophys. J. 79, 975-981 (2000).

23 Levicky, R. \& Horgan, A. Physicochemical perspectives on DNA microarray and biosensor technologies. Trends Biotechnol. 23, 143-149 (2005).
24 Pei, H., Li, F., Wan, Y., Wei, M., Liu, H. J., Su, Y., Chen, N., Huang, Q. \& Fan, C. H. Designed diblock oligonucleotide for the synthesis of spatially isolated and highly hybridizable functionalization of DNA-gold nanoparticle nanoconjugates. J. Am. Chem. Soc. 134, 11876-11879 (2012).

25 Leff, D. V., Brandt, L. \& Heath, J. R. Synthesis and characterization of hydrophobic, organically-soluble gold nanocrystals functionalized with primary amines. Langmuir 12, 4723-4730 (1996).

26 Walker, H. W. \& Grant, S. B. Conformaion of DNA block-copolymer molecules adsorbed on latex-particles as revealed by hydroxyl radical footpriting. Langmuir 11, 3772-3777 (1995).

27 Charreyre, M. T., Tcherkasskaya, O., Winnik, M. A., Hiver, A., Delair, T., Cros, P., Pichot, C. \& Mandrand, B. Fluorescence energy transfer study of the conformation of oligonucleotides covalently bound to polystyrene latex particles. Langmuir 13, 3103-3110 (1997).

28 Petrovykh, D. Y., Perez-Dieste, V., Opdahl, A., Kimura-Suda, H., Sullivan, J. M., Tarlov, M. J., Himpsel, F. J. \& Whitman, L. J. Nucleobase orientation and ordering in films of single-stranded DNA on gold. J. Am. Chem. Soc. 128, 2-3 (2006).

29 Lee, C. Y., Gong, P., Harbers, G. M., Grainger, D. W., Castner, D. G. \& Gamble, L. J. Surface coverage and structure of mixed DNA/alkylthiol monolayers on gold: characterization by XPS, NEXAFS, and fluorescence intensity measurements. Anal. Chem. 78, 3316-3325 (2006).

30 Liu, D. S., Cheng, E. J. \& Yang, Z. Q. A reversible pH-driven DNA nanoswitch array. J. Am. Chem. Soc. 128, 2067-2071 (2006).

31 Boozer, C., Chen, S. \& Jiang, S. Controlling DNA orientation on mixed ssDNA/OEG SAMs. Langmuir 22, 4694-4698 (2006).

32 Gong, P., Lee, C. Y., Gamble, L. J., Castner, D. G. \& Grainger, D. W. Hybridization behavior of mixed DNA/alkylthiol monolayers on gold: characterization by surface plasmon resonance and P-32 radiometric assay. Anal. Chem. 78, 3326-3334 (2006).

33 Zhang, J., Lao, R. J., Song, S. P., Yan, Z. Y. \& Fan, C. H. Design of an oligonucleotideincorporated nonfouling surface and its application in electrochemical dna sensors for highly sensitive and sequence-specific detection of target DNA. Anal. Chem. 80, 9029-9033 (2008)

34 Gong, P. \& Levicky, R. DNA surface hybridization regimes. Proc. Natl. Acad. Sci. USA 105, 5301-5306 (2008).

35 Hagan, M. F. \& Chakraborty, A. K. Hybridization dynamics of surface immobilized DNA. J. Chem. Phys. 120, 4958-4968 (2004).

36 Josephs, E. A. \& Ye, T. A single-molecule view of conformational switching of DNA tethered to a gold electrode. J. Am. Chem. Soc. 134, 10021-10030 (2012).

37 Fan, C. H., Plaxco, K. W. \& Heeger, A. J. Electrochemical interrogation of conformational changes as a reagentless method for the sequence-specific detection of DNA. Proc. Natl. Acad. Sci. USA 100, 9134-9137 (2003).

38 Liu, G., Wan, Y., Gau, V., Zhang, J., Wang, L. H., Song, S. P. \& Fan, C. H. An enzymebased E-DNA sensor for sequence-specific detection of femtomolar DNA targets. J. Am. Chem. Soc. 130, 6820-6825 (2008).

39 Xiao, Y., Qu, X. G., Plaxco, K. W. \& Heeger, A. J. Label-free electrochemical detection of DNA in blood serum via target-induced resolution of an electrode-bound DNA pseudoknot. J. Am. Chem. Soc. 129, 11896-11897 (2007).

40 Xiao, Y., Lou, X., Uzawa, T., Plakos, K. J., Plaxco, K. W. \& Soh, H. T. An electrochemical sensor for single nucleotide polymorphism detection in serum based on a triple-stem DNA probe. J. Am. Chem. Soc. 131, 15311-15316 (2009).

41 Xiao, Y., Rowe, A. A. \& Plaxco, K. W. Electrochemical detection of parts-per-billion lead via an electrode-bound DNAzyme assembly. J. Am. Chem. Soc. 129, 262-263 (2007).

42 Zuo, X. L., Song, S. P., Zhang, J., Pan, D., Wang, L. H. \& Fan, C. H. A target-responsive electrochemical aptamer switch (TREAS) for reagentless detection of nanomolar ATP. J. Am. Chem. Soc. 129, 1042-1043 (2007).

43 Lubin, A. A., Lai, R. Y., Baker, B. R., Heeger, A. J. \& Plaxco, K. W. Sequence-specific, electronic detection of oligonucleotides in blood, soil, and foodstuffs with the reagentless, reusable E-DNA sensor. Anal. Chem. 78, 5671-5677 (2006).

44 Lai, R. Y., Lagally, E. T., Lee, S.-H., Soh, H. T., Plaxco, K. W. \& Heeger, A. J. Rapid, sequence-specific detection of unpurified PCR amplicons via a reusable, electrochemical sensor. Proc. Natl. Acad. Sci. USA 103, 4017-4021 (2006).

45 Bockisch, B., Grunwald, T., Spillner, E. \& Bredehorst, R. Immobilized stem-loop structured probes as conformational switches for enzymatic detection of microbial $16 \mathrm{~S}$ rRNA. Nucleic Acids Res. 33, e101 (2005).

46 Wei, F., Wang, J. H., Liao, W., Zimmermann, B. G., Wong, D. T. \& Ho, C. M. Electrochemical detection of low-copy number salivary RNA based on specific signal amplification with a hairpin probe. Nucleic Acids Res. 36, e65 (2008).

47 Du, H., Disney, M. D., Miller, B. L. \& Krauss, T. D. Hybridization-based unquenching of DNA hairpins on Au surfaces: prototypical 'molecular beacon' biosensors. J. Am. Chem. Soc. 125, 4012-4013 (2003).

48 Du, H., Strohsahl, C. M., Camera, J., Miller, B. L. \& Krauss, T. D. Sensitivity and specificity of metal surface-immobilized 'molecular beacon' biosensors. J. Am. Chem. Soc. 127, 7932-7940 (2005).

49 Dubertret, B., Calame, M. \& Libchaber, A. J. Single-mismatch detection using goldquenched fluorescent oligonucleotides. Nat. Biotechnol. 19, 365-370 (2001).

50 Han, M. Y., Gao, X. H., Su, J. Z. \& Nie, S. Quantum-dot-tagged microbeads for multiplexed optical coding of biomolecules. Nat. Biotechnol. 19, 631-635 (2001).

51 Singh, A. K., Senapati, D., Wang, S. G., Griffin, J., Neely, A., Candice, P., Naylor, K. M., Varisli, B., Kalluri, J. R. \& Ray, P. C. Gold nanorod based selective identification of Escherichia coli bacteria using two-photon Rayleigh scattering spectroscopy. ACS Nano 3, 1906-1912 (2009). 
52 Song, S. P., Liang, Z. Q., Zhang, J., Wang, L. H., Li, G. X. \& Fan, C. H. Goldnanoparticle-based multicolor nanobeacons for sequence-specific DNA analysis. Angew. Chem. Int. Ed. 48, 8670-8674 (2009).

53 Jayagopal, A., Halfpenny, K. C., Perez, J. W. \& Wright, D. W. Hairpin DNAfunctionalized gold colloids for the imaging of mRNA in live cells. J. Am. Chem. Soc. 132, 9789-9796 (2010).

54 Xiao, Y., Lubin, A. A., Baker, B. R., Plaxco, K. W. \& Heeger, A. J. Single-step electronic detection of femtomolar DNA by target-induced strand displacement in an electrodebound duplex. Proc. Natl. Acad. Sci. USA 103, 16677-16680 (2006).

55 Huang, Y. C., Ge, B. X., Sen, D. \& Yu, H. Z. Immobilized DNA switches as electronic sensors for picomolar detection of plasma proteins. J. Am. Chem. Soc. 130, 8023-8029 (2008).

56 Huang, Y. C. \& Sen, D. A contractile electronic switch made of DNA. J. Am. Chem. Soc. 132, 2663-2671 (2010)

57 Zheng, D., Seferos, D. S., Giljohann, D. A., Patel, P. C. \& Mirkin, C. A. Aptamer nano-flares for molecular detection in living cells. Nano Lett. 9, 3258-3261 (2009).

58 Pei, H., Lu, N., Wen, Y. L., Song, S. P., Liu, Y., Yan, H. \& Fan, C. H. A dna nanostructure-based biomolecular probe carrier platform for electrochemical biosensing. Adv. Mater. 22, 4754-4758 (2010).

59 Wen, Y. L., Pei, H., Wan, Y., Su, Y., Huang, Q., Song, S. P. \& Fan, C. H. DNA nanostructure-decorated surfaces for enhanced aptamer-target binding and electrochemical cocaine sensors. Anal. Chem. 83, 7418-7423 (2011).

60 Pei, H., Wan, Y., Li, J., Hu, H. Y., Su, Y., Huang, Q. \& Fan, C. H. Regenerable electrochemical immunological sensing at DNA nanostructure-decorated gold surfaces. Chem. Commun. 47, 6254-6256 (2011).

61 Wen, Y., Pei, H., Shen, Y., Xi, J. J., Lin, M. H., Lu, N., Shen, X. Z., Li, J. \& Fan, C. H. DNA nanostructure-based interfacial engineering for PCR-free ultrasensitive electrochemical analysis of microRNA. Sci. Rep. 2, 867 (2012).

62 Schlapak, R., Danzberger, J., Armitage, D., Morgan, D., Ebner, A., Hinterdorfer, P., Pollheimer, P., Gruber, H. J., Schaffler, F. \& Howorka, S. Nanoscale DNA tetrahedra improve biomolecular recognition on patterned surfaces. Small 8, 89-97 (2012).

63 Squires, T. M., Messinger, R. J. \& Manalis, S. R. Making it stick: convection, reaction and diffusion in surface-based biosensors. Nat. Biotechnol. 26, 417-426 (2008)

64 Mitchell, N., Schlapak, R., Kastner, M., Armitage, D., Chrzanowski, W., Riener, J., Hinterdorfer, P. Ebner, A. \& Howorka, S. A DNA nanostructure for the functional assembly of chemical groups with tunable stoichiometry and defined nanoscale geometry. Angew. Chem. Int. Ed. 48, 525-527 (2009).

$65 \mathrm{Ge}, \mathrm{Z} . \mathrm{L}$, Pei, $\mathrm{H}$., Wang, L. H., Song, S. P. \& Fan, C. H. Electrochemical single nucleotide polymorphisms genotyping on surface immobilized three-dimensional branched DNA nanostructure. Sci. China-Chem. 54, 1273-1276 (2011).

$66 \mathrm{Bu}$, N. N., Tang, C. X., He, X. W. \& Yin, X. B. Tetrahedron-structured DNA and functional oligonucleotide for construction of an electrochemical DNA-based biosensor. Chem. Commun. 47, 7689-7691 (2011).

67 Lu, N., Pei, H., Ge, Z. L., Simmons, C. R., Yan, H. \& Fan, C. H. Charge transport within a three-dimensional DNA nanostructure framework. J. Am. Chem. Soc. 134 13148-13151 (2012).

68 Pei, H., Liang, L., Yao, G. B., Li, J., Huang, Q. \& Fan, C. H. Reconfigurable three dimensional DNA nanostructures for the construction of intracellular logic sensors. Angew. Chem. Int. Ed. 51, 9020-9024 (2012).

69 Ke, Y. G., Lindsay, S., Chang, Y., Liu, Y. \& Yan, H. Self-assembled water-soluble nucleic acid probe tiles for label-free RNA hybridization assays. Science 319, 180-183 (2008).

70 Rothemund, P. W. K. Folding DNA to create nanoscale shapes and patterns. Nature 440, 297-302 (2006).

71 Zhang, Z., Wang, Y., Fan, C. H., Li, C., Li, Y., Qian, L. L., Fu, Y. M., Shi, Y. Y., Hu, J. \& $\mathrm{He}, \mathrm{L}$. Asymmetric DNA origami for spatially addressable and index-free solution-phase DNA chips. Adv. Mater. 22, 2672-2675 (2010).

72 Zhang, Z., Zeng, D. D., Ma, H. W., Feng, G. Y., Hu, J., He, L., Li, C. \& Fan, C. H. A DNA-origami chip platform for label-free SNP genotyping using toehold-mediated strand displacement. Small 6, 1854-1858 (2010).

73 Subramanian, H. K. K., Chakraborty, B., Sha, R. \& Seeman, N. C. The label-free unambiguous detection and symbolic display of single nucleotide polymorphisms on DNA origami. Nano Lett. 11, 910-913 (2011).

74 Liu, D. S., Cheng, E. J. \& Yang, Z. Q. DNA-based switchable devices and materials. NPG Asia Mater. 3, 109-114 (2011).

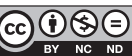

This work is licensed under a Creative Commons Attribution-NonCommercial-NoDerivs 3.0 Unported License. To view a copy of this license, visit http://creativecommons. org/licenses/by-nc-nd/3.0/
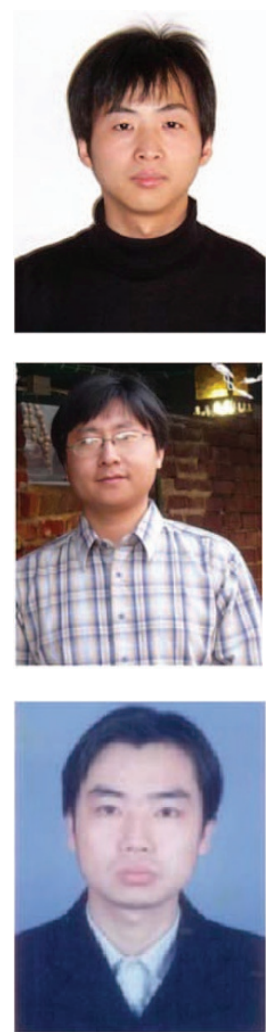

Hao Pei was born in Hubei, China, in 1984. He received his BS degree (2006) in chemistry from the Nanjing University, and then obtained his $\mathrm{PhD}$ under the supervision of Professor Chunhai Fan at the Shanghai Institute of Applied Physics (SINAP), Chinese Academy of Sciences. In 2012, he joined the Division of Physical Biology, SINAP as an assistant investigator. His research interests include DNA as construction materials, and exploring potential applications such as biosensing, biocomputing and nanophonics.

Xiaolei Zuo obtained his BS degree from the Department of Chemistry and Chemical Engineering at the Central South University in 2002 and his PhD at the Shanghai Institute of Applied Physics (SINAP) in 2008. After his postdoctoral research at the University of California at Santa Barbara and the Los Alamos National Laboratory, he joined the faculty at SINAP, Chinese Academy of Sciences (CAS) in 2012. He is now a Professor and funded by One Hundred Talent Project of CAS. Professor Zuo published his scientific papers in peer-reviewed journals, including Chemical Reviews, PNAS and JACS.

Dun Pan obtained his BS degree from the Department of Applied Chemistry at the Beijing University of Chemical Technology and his $\mathrm{PhD}$ at the Technical Institute of Physics and Chemistry of Chinese Academy of Sciences (CAS) in 2005. After his postdoctoral research at the Shanghai Institute of Applied Physics (SINAP), he joined the faculty at SINAP, CAS in 2008. He is now an associate professor. Professor Pan published his scientific papers in peer-reviewed journals. His research interests include biosensors and nanobiology. 


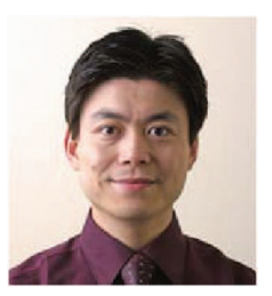

Jiye Shi obtained his BS degree from the Department of Biochemistry at Nanjing University in 1998, his PhD from the Department of Biochemistry at Cambridge University in 2001 and his MBA from the Simon Business School at the University of Rochester in 2009. He joined the pharmaceutical industry in 2001 and is now Director of Computational Structural Biology at UCB, a global biopharmaceutical company with $€ 3.5$ billion in revenue. He also serves on both the executive management committee and the scientific management committee of the Industrial Doctorate Center at Oxford University, and holds guest professorship at the Shanghai Institute of Applied Physics, Chinese Academy of Sciences.

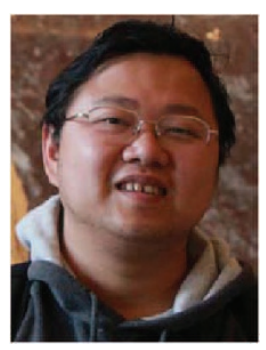

Qing Huang obtained his BS degree from the Department of Biochemistry at Nanjing University in 1996 and PhD from the School of Life Sciences at Sichuang University in 2003. After his postdoctoral research in Louisiana State University, he joined the faculty of Shanghai Institute of Applied Physics (SINAP), Chinese Academy of Sciences, in 2003. He is now Professor and Deputy Chief of the Division of Physical Biology at SINAP.

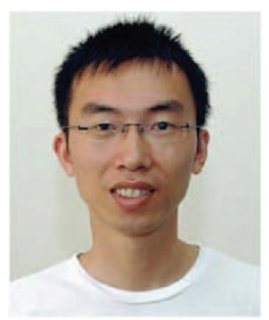

Chunhai Fan obtained his BS degree and PhD from the Department of Biochemistry at Nanjing University in 1996 and 2000. After his postdoctoral research at the University of California, Santa Barbara, he joined the faculty at the Shanghai Institute of Applied Physics (SINAP), Chinese Academy of Sciences, in 2004. He is now Professor and Chief of the Division of Physical Biology and the Bioimaging Center at SINAP. He is also the associate editor of ACS Applied Materials and Interfaces. Dr Fan has published over 200 papers in peerreviewed journals. 